\title{
Postpartum depression and traditional postpartum care in China: Role of Zuoyuezi
}

\author{
Ellen Y. Wan ${ }^{\mathrm{a}, \mathrm{b}}$, Cheryl A. Moyer ${ }^{\mathrm{c}, \mathrm{d}, *}$, Siobán D. Harlow ${ }^{\mathrm{a}}$, Zitian Fan ${ }^{\mathrm{e}}$, Yan Jie ${ }^{\mathrm{e}}$, Huixia Yang e,* \\ a Department of Epidemiology, University of Michigan School of Public Health, Ann Arbor, MI, USA \\ b Centers for Disease Control and Prevention, Atlanta, GA, USA \\ c Global REACH, University of Michigan Medical School, Ann Arbor, MI, USA \\ d Department of Medical Education, University of Michigan Medical School, Ann Arbor, MI, USA \\ e Department of Obstetrics and Gynecology, Peking University First Hospital, Beijing, China
}

\section{A R T I C L E I N F O}

\section{Article history:}

Received 16 July 2008

Received in revised form 17 October 2008

Accepted 21 October 2008

\section{Keywords:}

China

Postnatal depression

Postpartum care

Postpartum depression

Urban

Zuoyuezi

\begin{abstract}
A B S T R A C T
Objective: To determine the relationship between the traditional Chinese practice of postpartum care, known as zuoyuezi, and postpartum depression (PPD) in China. Methods: A total of 342 Chinese women were surveyed 6- to 8-weeks post partum using the Edinburgh Postnatal Depression Scale (EPDS) and items assessing sociodemographics, health history, peripartum experiences, zuoyuezi, and social support. Results: Prevalence of PPD was 15.5\% (EPDS cutoff $\geq 13$ ). PPD was associated with lower income, difficult pregnancy experience, poor infant health status, not attending childbirth classes, and low spousal involvement before and after delivery. Among the $96 \%$ of women who practiced zuoyuezi, those for whom the caregiver was her mother-in-law or who perceived zuoyuezi as unhelpful had twice the odds of PPD. Conclusion: These data highlight the importance of the peripartum experience in assessing PPD risk. Zuoyuezi is still commonly practiced in urban China, and further research is needed to explore its role in the potential prevention of PPD. (c) 2008 Published by Elsevier Ireland Ltd. on behalf of International Federation of Gynecology and Obstetrics.
\end{abstract}

\section{Introduction}

Postpartum depression (PPD) affects $10 \%$ to $15 \%$ of postpartum women worldwide [1,2] with even greater rates among women of low socioeconomic status [2] and those who report psychological stressors [3]. Although potential risk factors have been identified [2-5], little research has focused on traditional postpartum practices.

Postpartum practices vary across cultures, but most include a designated period of extra care for the mother [6]. In China, this practice is called zuoyuezi, or "doing the month" [7]. The new mother stays at home for one month after childbirth, during which she is bound to a set of strict guidelines to restore the energy balance within the body and observe taboos that, if broken, are believed to bring misfortune and ill health [7]. Researchers have identified 4 key components to zuoyuezi: (1) to have the woman's mother, mother-in-law, a paid nurse, and/or partner help her recuperate, adjust to motherhood, and free her from domestic duties; (2) to avoid physical contact with anything cold (eg, drafts, cold winds, cold water, ice, cold foods); (3) to brush one's teeth and bathe with warm, boiled water; and (4) to maintain a balance between hot and cold intake of food [8].

Recent studies have examined the relationship between zuoyuezi and PPD among Chinese women in Hong Kong and Taiwan. In Taiwan,

\footnotetext{
* Corresponding authors. Moyer is to be contacted at Global REACH, 717 E. Huron, Suite 1E, Ann Arbor, MI 48104, USA. Tel.: +1 734998 6827; fax: +1 734998 6825. Yang, Department of OB/GYN, Peking University First Hospital, No. 8 XiShiKu St. XiCheng District, Beijing, China. Tel.: +86 106655 1122; fax: +8610 66186087.

E-mail addresses: camoyer@umich.edu (C.A. Moyer), yanghuixia@bjmu.edu.cn (H. Yang).
}

postpartum women who received care from their own mothers exhibited fewer depressive symptoms [9]. Increased social support was associated with reduced stress levels and better health status [10]. In Hong Kong, zuoyuezi was associated with better social support and lower risk of PPD [11] while conflict with the mother-in-law was predictive of PPD.

Few studies on PPD have been conducted in China. A MEDLINE search found abstracts of Chinese articles from Beijing [12] and several provinces [13-15]. All used convenience samples from their respective clinics and nonvalidated measures of PPD to estimate PPD prevalence. A separate study used the validated Edinburgh Postnatal Depression Scale (EPDS) [16] and reported that $17.3 \%$ of postpartum women had PPD, with the strongest risk factor being the birth of a female infant [17].

The current study aimed to estimate the prevalence of PPD among new mothers in Beijing, China, and to evaluate potential risk factors, particularly factors involving social support and the practice of zuoyuezi.

\section{Materials and methods}

The study protocol was reviewed and approved by institutional review boards in China and the United States. This cross-sectional study was conducted between May and July 2006 in the obstetric outpatient clinic at Peking University First Hospital, one of the largest academic medical centers in Beijing. This hospital serves both public and private patients from in and around Beijing. The clinic dedicates one day per week to postpartum mothers.

A convenience sample of all postpartum women aged 18 years or older arriving at the clinic for their 6- to 8-week postpartum follow-up 
Table 1

Crude associations between sociodemographic factors, health history, and experiences with the recent pregnancy and delivery and EPDS scores $(n=342)$

\begin{tabular}{|c|c|c|c|c|c|c|}
\hline \multirow[t]{2}{*}{ Characteristics } & \multicolumn{2}{|l|}{ Total } & \multicolumn{3}{|c|}{ EPDS $\geq 13$} & \multirow[b]{2}{*}{$95 \% \mathrm{CI}$} \\
\hline & No. & $\%$ & No. & $\%$ & OR & \\
\hline \multicolumn{7}{|c|}{ Demographic characteristics } \\
\hline \multicolumn{7}{|c|}{ Age, y } \\
\hline $15-24$ & 11 & 3.2 & 4 & 36.4 & 3.62 & $(0.81-16.22)$ \\
\hline $25-34$ & 287 & 83.9 & 43 & 15.0 & 1.17 & $(0.45-2.80)$ \\
\hline $35-44$ & 42 & 12.3 & 6 & 14.3 & 1.00 & \\
\hline Missing & 2 & & & & & \\
\hline \multicolumn{7}{|l|}{ From Beijing } \\
\hline Yes & 201 & 59.1 & 37 & 18.4 & 1.73 & $(0.92-3.26)$ \\
\hline No & 139 & 40.9 & 16 & 11.5 & 1.00 & \\
\hline Missing & 2 & & & & & \\
\hline \multicolumn{7}{|l|}{ Ethnicity } \\
\hline Han & 324 & 94.7 & 51 & 15.7 & 1.49 & $(0.33-6.69)$ \\
\hline Non-Han & 18 & 5.3 & 2 & 11.1 & 1.00 & \\
\hline Education & & & & & & \\
\hline Less than college & 100 & 29.2 & 15 & 15.0 & 1.12 & $(0.55-2.27)$ \\
\hline College graduate & 161 & 47.1 & 22 & 13.7 & 1.00 & \\
\hline More than college & 81 & 23.7 & 16 & 19.8 & 1.56 & $(0.77-3.16)$ \\
\hline Income (monthly, US & & & & & & \\
\hline$<325$ & 75 & 22.1 & 16 & 21.3 & 2.08 & $(1.02-4.25)$ \\
\hline $325-625$ & 85 & 25.0 & 16 & 18.8 & 1.78 & $(0.88-3.61)$ \\
\hline$>625$ & 180 & 52.9 & 21 & 11.7 & 1.00 & \\
\hline Missing & 2 & & & & & \\
\hline Car owner & & & & & & \\
\hline Yes & 157 & 46.0 & 18 & 11.5 & 1.00 & \\
\hline No & 184 & 54.0 & 35 & 19.0 & 1.81 & $(0.98-3.35)$ \\
\hline Missing & 1 & & & & & \\
\hline Have health insuranc & & & & & & \\
\hline Yes & 254 & 75.4 & 38 & 15.0 & 1.00 & \\
\hline No & 83 & 24.6 & 14 & 16.9 & 1.15 & $(0.59-2.25)$ \\
\hline Missing & 5 & & & & & \\
\hline General health history & & & & & & \\
\hline Previous health issue & & & & & & \\
\hline Yes & 36 & 10.6 & 4 & 11.1 & 1.00 & \\
\hline No & 304 & 89.4 & 49 & 16.1 & 1.54 & $(0.52-4.54)$ \\
\hline Missing & 2 & & & & & \\
\hline Ever had emotional i & & & & & & \\
\hline Yes & 15 & 4.4 & 4 & 26.7 & 2.06 & $(0.63-6.74)$ \\
\hline No & 327 & 95.6 & 49 & 15.0 & 1.00 & \\
\hline Pregnancy history & & & & & & \\
\hline Gravidity & & & & & & \\
\hline 1 & 159 & 47.8 & 29 & 18.2 & 1.56 & $(0.78-3.11)$ \\
\hline 2 & 103 & 30.9 & 13 & 12.6 & 1.00 & \\
\hline$>2$ & 71 & 21.3 & 10 & 14.1 & 1.15 & $(0.48-2.75)$ \\
\hline Missing & 9 & & & & & \\
\hline Previous abortion & & & & & & \\
\hline Yes & 172 & 50.7 & 22 & 12.79 & 1.00 & \\
\hline No & 167 & 49.3 & 31 & 18.56 & 1.55 & $(0.86-2.81)$ \\
\hline Missing & 3 & & & & & \\
\hline Pregnancy experienc & & & & & & \\
\hline Very easy & 82 & 24.3 & 5 & 6.1 & 1.00 & \\
\hline Somewhat easy & 177 & 52.4 & 28 & 15.8 & 2.12 & $(0.89-5.07)$ \\
\hline Difficult & 79 & 23.4 & 18 & 22.8 & 3.33 & $(1.31-8.48)$ \\
\hline Missing & 4 & & & & & \\
\hline Pregnancy complicat & & & & & & \\
\hline Yes & 69 & 20.3 & 14 & 20.3 & 1.51 & $(0.77-2.98)$ \\
\hline No & 271 & 79.7 & 39 & 14.4 & 1.00 & \\
\hline Missing & 2 & & & & & \\
\hline Childbirth experience & & & & & & \\
\hline Gestational age, wk & & & & & & \\
\hline$<37$ & 17 & 5.0 & 4 & 23.5 & 1.84 & $(0.58-5.89)$ \\
\hline$\geq 37$ & 321 & 95.0 & 46 & 14.3 & 1.00 & \\
\hline Missing & 4 & & & & & \\
\hline Delivery experience & & & & & & \\
\hline Excellent & 92 & 27.8 & 9 & 9.8 & 1.00 & \\
\hline Fair-Good & 181 & 54.7 & 26 & 14.4 & 0.98 & $(0.50-1.96)$ \\
\hline Poor & 58 & 17.5 & 12 & 20.7 & 1.53 & $(0.66-3.54)$ \\
\hline Missing & 11 & & & & & \\
\hline Birth weight, $g$ & & & & & & \\
\hline$<2500$ & 11 & 3.2 & 2 & 18.2 & 1.22 & $(0.26-5.79)$ \\
\hline$\geq 2500$ & 330 & 96.8 & 51 & 15.5 & 1.00 & \\
\hline Missing & 1 & & & & & \\
\hline Infant health status & & & & & & \\
\hline Excellent & 226 & 66.5 & 30 & 13.3 & 1.00 & \\
\hline Good & 84 & 24.7 & 14 & 16.7 & 1.32 & $(0.66-2.63)$ \\
\hline
\end{tabular}

Table 1 (continued)

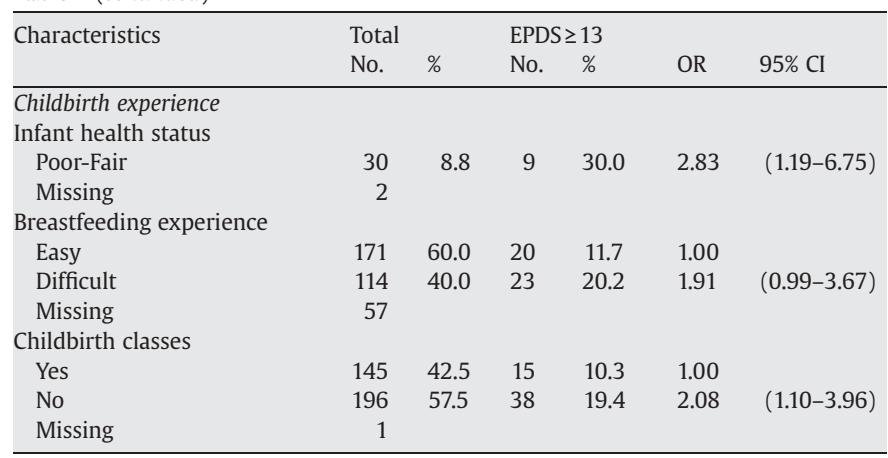

Abbreviation: EDPS, Edinburgh Postnatal Depression Scale.

visit were eligible to participate. Women were approached in the waiting room by a trained, bilingual research assistant and asked to participate. The goals of the study were described, and women were taken through an informed consent document. Women who agreed to participate completed the self-administered, paper-and-pencil questionnaire in a private room either before or after seeing the physician (post hoc statistical analysis revealed no significant difference in EPDS scores based on timing of instrument completion). No personal identifiers were collected.

The study instrument included the EPDS and items designed to assess sociodemographic characteristics, work history, health history, and the experiences of the recent pregnancy, childbirth, zuoyuezi, and social support. The questionnaire was created in English and then translated into Mandarin Chinese. The Mandarin Chinese version was subsequently checked with the English version for accuracy of translation.

The questionnaire assessed age, ethnicity, place of origin, urbanicity of residence, education, income, car ownership, and health insurance. For analysis purposes, monthly income was categorized in US dollars as less than $\$ 325, \$ 325-\$ 625$, and more than $\$ 625$. The average income across all sectors in Beijing is approximately $\$ 2000$, or 16,000 yuan per month [18]. The initial categories included income ranges exceeding 20,000 yuan per month, but the data reflected a skew toward lower reported incomes and categories were consolidated appropriately. To assess health history, women were asked whether they had any ongoing health issues unrelated to pregnancy about which they had seen a physician. History of mental illness was positive if women indicated they had ever consulted a healthcare provider for help with emotional issues or taken prescription or traditional Chinese medicines for emotional issues.

The instrument also assessed gravidity, previous abortion history, quality of recent pregnancy experience, and the presence of any pregnancy complications. Women were asked to rate their recent pregnancy experience on a scale of 1 to 5 (very difficult to very easy). Parturition experience was described by the length of labor, delivery type, the use of pain management medications, and care during delivery. Women rated their delivery experience on a scale of 1 to 10 . Answers were categorized as poor (1 to 4 ), fair-good (5 to 8), and excellent (9-10). Women were also asked whether they took childbirthing classes before delivery, or whether it took more than one year to conceive. Gestational age, birth weight, neonatal health status, and breastfeeding experience (easy/difficult) were also recorded. Neonatal health status was measured on a Likert scale with 1 indicating very poor health and 5 indicating excellent health. A rating of 3 or less was considered to be poor health status.

Women were asked if they practiced zuoyuezi, for how many weeks, and who primarily provided the care (the mother, mother-in-law, or other). Women rated helpfulness of zuoyuezi on a scale of 1 to 5 , with 1 being not helpful at all and 5 being extremely helpful. This scale was dichotomized for analysis with a rating of 3 or less considered to be not helpful. 
Table 2

Crude associations between social support characteristics $(n=342)$ and zuoyuezirelated characteristics $(n=327)$ and EPDS scores

\begin{tabular}{|c|c|c|c|c|c|c|}
\hline & \multicolumn{2}{|c|}{ Total } & \multicolumn{4}{|c|}{$E P D S \geq 13$} \\
\hline & No. & $\%$ & No. & $\%$ & OR & $95 \% \mathrm{CI}$ \\
\hline \multicolumn{7}{|c|}{ Zuoyuezi caregiver $(\mathrm{n}=327)$} \\
\hline Mother only & 156 & 50.8 & 20 & 12.8 & 1.00 & \\
\hline Mother-in-law & 110 & 35.8 & 24 & 21.8 & 2.06 & $(1.08-3.92)$ \\
\hline Other only & 41 & 13.4 & 5 & 12.2 & 1.03 & $(0.36-2.90)$ \\
\hline Missing & 20 & & & & & \\
\hline \multicolumn{7}{|c|}{ Zuoyuezi was helpful ( $\mathrm{n}=327)$} \\
\hline Yes & 178 & 54.9 & 19 & 10.7 & 1.00 & \\
\hline No & 146 & 45.1 & 31 & 21.2 & 2.26 & $(1.21-4.19)$ \\
\hline Missing & 3 & & & & & \\
\hline \multicolumn{7}{|c|}{ Relationship with parents } \\
\hline Good-Excellent & 308 & 90.3 & 44 & 14.3 & 1.00 & \\
\hline Poor-Fair & 33 & 9.7 & 9 & 27.3 & 2.25 & $(0.98-5.16)$ \\
\hline Missing & 1 & & & & & \\
\hline \multicolumn{7}{|c|}{ Relationship with parents-in-law } \\
\hline Good-Excellent & 250 & 73.5 & 33 & 13.2 & 1.00 & \\
\hline Poor-Fair & 90 & 26.5 & 19 & 21.1 & 1.76 & $(0.94-3.29)$ \\
\hline Missing & 2 & & & & & \\
\hline \multicolumn{7}{|l|}{ Marital satisfaction } \\
\hline Very satisfied & 233 & 70.2 & 29 & 12.5 & 1.00 & \\
\hline Satisfied & 71 & 21.4 & 14 & 19.7 & 1.62 & $(0.81-3.24)$ \\
\hline Not satisfied & 28 & 8.4 & 7 & 25.0 & 2.20 & $(0.87-5.59)$ \\
\hline Missing & 10 & & & & & \\
\hline \multicolumn{7}{|c|}{ Spousal involvement during pregnancy } \\
\hline Involved & 268 & 78.4 & 36 & 13.4 & 1.00 & \\
\hline Not involved & 74 & 21.6 & 17 & 23.0 & 1.92 & $(1.01-3.67)$ \\
\hline \multicolumn{7}{|c|}{ Spousal involvement after delivery } \\
\hline Involved & 292 & 85.6 & 39 & 13.4 & 1.00 & \\
\hline Not involved & 49 & 14.4 & 14 & 28.6 & 2.60 & $(1.28-5.26)$ \\
\hline Missing & 1 & & & & & \\
\hline
\end{tabular}

Abbreviation: EDPS, Edinburgh Postnatal Depression Scale.

Women were asked to rate the quality of their relationships with their parents and in-laws on Likert scales of 1 (very poor) to 5 (excellent). Marital satisfaction was rated with 1 representing least satisfied and 5 representing most satisfied. The husband's involvement both during pregnancy and after delivery were rated between 1 for not involved at all and 5 for extremely involved. Each scale was dichotomized for analysis with 3 or less indicating poor, not satisfied, or not involved, respectively.

The validated Mandarin Chinese version of the EPDS was used to measure PPD [15]. As originally validated, the Chinese version of the EPDS used a cutoff score of 10 or more to suggest PPD [16]. A subsequent validation within a Taiwanese Chinese population used a cutoff of 13 or more [19], similar to the original English version of the EPDS. Since the EPDS has not been validated in the mainland Chinese population, analyses were run using both cutoff scores in the present study. As results were similar, results are presented for the 13 or more cutoff score.

SAS version 9.1 (SAS, Cary, NC, USA) was used for all analyses. Descriptive statistics for all variables were examined. Bivariate associations between the outcome of EPDS scores and each variable were assessed by calculating crude odds ratios and 95\% confidence intervals. Multiple logistic regression models were built first for all women in the study sample and then for the subset of women who practiced zuoyuezi. Variables significantly associated with EPDS scores in univariate analysis were chosen for inclusion in the multiple logistic regression.

\section{Results}

Of the 471 postpartum women who attended clinic on the days of data collection and were thus eligible to participate in the study, 395 (77\%) women were asked to participate. Ninety-two percent (365) agreed to participate and returned a questionnaire, and 342 of those questionnaires (93.7\% of completed surveys) were completed sufficiently to be included in the analysis. The remaining women were excluded.
Table 1 presents the sociodemographic characteristics of the 342 participants. The mean age was $30 \pm 3.4$ years, $71 \%$ had at least a college degree, and more than half (53\%) lived in households earning more than US $\$ 625$ monthly. Few women reported previously existing health issues, and only $4 \%$ reported having sought care for emotional issues. The recent pregnancy was the first for $48 \%$ of the women. More than $50 \%$ had previously had an abortion. During the recent pregnancy, 20\% experienced complications, and $23 \%$ reported having a difficult pregnancy. Twenty-eight percent reported an excellent delivery experience, $55 \%$ fair to good, and $18 \%$ a poor experience. Forty-three percent of mothers had taken childbirth classes before delivery.

Table 2 provides information on women's social support and zuoyuezi characteristics. Zuoyuezi was practiced by 327 (96\%) of the women. For $51 \%$ of these women, care during the period of zuoyuezi was provided only by their mothers. Thirty-six percent received care from their mother-in-law, and 13\% from someone else. Of the women who practiced zuoyuezi, 55\% reported that the practice was useful.

Scores on the EPDS among all women ranged from 0 to 27 (mean score, $8.4 \pm 4.0$ ), with $15.5 \%$ screening positive for PPD. Higher odds of PPD were associated with lower income, more difficult pregnancy experience, poor infant health status, and not attending childbirth classes (Table 1). In addition, women who reported a lack of spousal involvement before and after delivery had 1.9 and 2.6 times greater odds of having PPD than women who reported higher levels of spousal involvement (Table 2). Sex of the neonate, feeding method, having had difficulty conceiving, and having a nanny were not associated with having PPD (data not shown).

The practice of zuoyuezi itself was not associated with PPD (OR 0.92; 95\% CI, 0.20- 4.25), but women whose zuoyuezi caregiver was their mother-in-law (35.8\%) and women who believed that zuoyuezi was not helpful (45.1\%) had twice the odds of PPD (Table 2).

In the adjusted analysis for all women $(n=342)$, car ownership, pregnancy experience, spousal involvement during pregnancy, childbirth classes, and infant health status remained independent risk factors for having postpartum depressive symptoms (Table 3). A difficult pregnancy experience and poor infant health status were significantly associated with more than a 3 -fold increase in odds of PPD, while not having taken childbirth classes doubled the odds of having PPD. When spousal involvement during pregnancy was replaced with involvement following delivery, a stronger association was found (OR 2.33; 95\% CI, 1.10-4.93). When substituting income for car ownership in the model, all associations were attenuated, and income did not contribute significantly to the adjusted model.

Table 3

Adjusted odds ratios using multiple variable logistic regression for the main effects model on the outcome of EDPS score $\geq 13$, describing all women in the sample and women who practiced zuoyuezi

\begin{tabular}{|c|c|c|}
\hline & $\begin{array}{l}\text { All women } \\
(\mathrm{n}=342)\end{array}$ & $\begin{array}{l}\text { Women who practiced } \\
\text { zuoyuezi }(n=327)\end{array}$ \\
\hline & OR (95\% CI) & OR (95\% CI) \\
\hline Not a car owner & $1.88(0.99-3.59)$ & $1.92(0.97-3.83)$ \\
\hline \multicolumn{3}{|l|}{ Pregnancy experience } \\
\hline Very easy & 1 & 1 \\
\hline Somewhat easy & $2.31(0.94-5.64)$ & $2.71(1.03-7.14)$ \\
\hline Difficult & $3.61(1.38-9.45)$ & $4.10(1.45-11.56)$ \\
\hline Spousal involvement during pregnancy & $1.96(0.99-3.85)$ & $1.58(0.75-3.31)$ \\
\hline No childbirth classes & $2.30(1.18-4.49)$ & $1.90(0.95-3.79)$ \\
\hline \multicolumn{3}{|l|}{ Infant health status } \\
\hline Excellent & 1 & 1 \\
\hline Good & $1.13(0.55-2.33)$ & $1.10(0.51-2.37)$ \\
\hline Poor-Fair & $3.09(1.22-7.82)$ & $2.68(1.01-7.14)$ \\
\hline \multicolumn{3}{|l|}{ Zuoyuezi caregiver $(\mathrm{n}=327)$} \\
\hline Mother only & & 1 \\
\hline Mother-in-law & & $2.14(1.08-4.26)$ \\
\hline Other only & & $1.10(0.36-3.31)$ \\
\hline Zuoyuezi not helpful & & $2.16(1.12-4.16)$ \\
\hline
\end{tabular}

Abbreviation: EDPS, Edinburgh Postnatal Depression Scale. 
For women who practiced zuoyuezi, both care by the mother-in-law and a perceived unhelpfulness of practicing zuoyuezi were associated with a 2 -fold increase in odds of postpartum depressive symptoms, independent of other risk factors.

\section{Discussion}

Using the EPDS, an estimated $15.5 \%$ of postpartum women at Peking University First Hospital were determined to have PPD. Zuoyuezi is still a common practice that is well-known and expected for Chinese women following childbirth. More than 95\% of the women in the sample practiced zuoyuezi, which limited the power to assess whether it was helpful itself in reducing risk of PPD. However, among women who practiced zuoyuezi, care given by the mother-in-law and the perception that the practice of zuoyuezi was not helpful were each associated with more than a 2-fold increase in the odds of PPD. The most important predictors of PPD were having a difficult pregnancy experience, poor infant health status, lack of spousal involvement during pregnancy, and not taking childbirth classes. There was no evidence to suggest that the delivery experience itself or the sex of the neonate was related to PPD in this population.

The finding that having the mother-in-law as zuoyuezi caregiver was a risk factor for PPD supports previous research from Taiwan and Hong Kong [10-12]. Previous studies have found that mother-in-law conflict is a significant stressor for married Chinese women and has been implicated in one-third of suicide attempts by young women in rural China [20].

Although a negative perception of the helpfulness of zuoyuezi was associated with a doubling in odds of PPD, this cross-sectional study cannot clarify the temporal nature of the relationship. The woman's mental state at the point of assessment may have influenced her selfreported experience. Also, helpfulness of zuoyuezi was assessed with only one question. Further research is needed that expands upon our understanding of how and why women perceive zuoyuezi to be helpful or not. Information about the quality or type of social support provided was not available. Distinctions between perceived and actual social support provided during zuoyuezi, as well as distinctions among the types of support provided require further examination to better understand the role it has in providing support and potentially preventing PPD.

The association between PPD and spousal involvement, both during pregnancy and after delivery, was significant, yet weaker than expected. Previous research has supported the importance of partner support for positive pregnancy outcomes [21]. When the characteristics of zuoyuezi were considered, the association weakened. Therefore, social support provided by the mother or mother-in-law during zuoyuezi may be equally or even more important than the husband's support in this population. However, zuoyuezi is often provided by multiple caregivers, including parents, in-laws and the husband together, and these effects could not be differentiated within the present study.

Two factors strongly associated with PPD were self-reported ease of the pregnancy experience and infant health status. However, both associations may be affected by recall bias since women were asked to rate them 6- to 8-weeks postpartum.

Taking childbirth classes during pregnancy was also associated with lower odds of PPD. Classes may be a marker for socioeconomic status, since they require out-of-pocket payment. Consistent with previous research, it was observed that lower income can be associated with an increased risk of PPD [22]. However, classes may better prepare women for childbirth and the postpartum period. Future research should examine the content of these classes and the impact of frequency, length, and timing of classes during pregnancy on PPD.

No aspect of the delivery experience was associated with PPD. This finding is consistent with previous research [23] and reinforces the importance of the prenatal and immediate postpartum period in defining women's risk of PPD.
An unexpected finding was the lack of association between infant gender and PPD risk. Previous research in China found that women who bore female infants were nearly 3 times more likely to suffer PPD than mothers of male children [17]. The one-child policy in China and the traditional preference for boys over girls is well known. However, urban women may be happy with a child that is healthy, regardless of its gender.

Disagreement persists about the optimal EPDS cutoff point for PPD, and had the lower cutoff point been used (EPDS $\geq 10$ ), more than $30 \%$ of the sample would have been categorized as having PPD. Use of the $\geq 13$ cutoff point yields a more conservative estimate. Given that previous mental health issues are a strong risk factor for PPD $[2,4,5]$ it was surprising that only $4 \%$ of these women reported ever having sought care for emotional issues. Chinese patients may experience mental health conditions somatically and perceive their ailments as physical rather than mental illness [24]. Therefore, Chinese patients may be less likely to consult mental health professionals [25]. It also may reflect social norms that value stoicism. Alternative strategies for assessment of mental health history in this population are needed.

Finally, these findings are not representative of all Chinese women, since the sample was urban and well educated. In addition, although incomes varied across the sample and appeared to be lower than citywide averages, $75 \%$ of the women had health insurance, compared with the urban average of 39\% [26]. Nonetheless, the present study adds to the literature on PPD in mainland China.

In conclusion, these results suggest that many factors impact the likelihood of a woman developing PPD. While some, such as income, may not be easy to change, others can be addressed through thoughtful intervention or public policy changes. For example, childbirth classes could be subsidized or provided free by the hospital. Spouses-and mothers-in-law-could be educated regarding the types of support that women find useful. Further studies are needed to deconstruct the practice of zuoyuezi and examine the factors that contribute to maternal mental health outcomes in China.

\section{Acknowledgment}

National Institutes of Health funding was used for this study (T37 MD001425-08).

\section{References}

[1] Mallikarjun PK, Oyebode F. Prevention of postnatal depression. J R Soc Health 2005;125(5):221-6.

[2] Perfetti J, Clark R, Fillmore CM. Postpartum depression: identification, screening, and treatment. WMJ 2004;103(6):56-63.

[3] Fontaine KR, Jones LC. Self-esteem, optimism, and postpartum depression. J Clin Psychol 1997;53(1):59-63.

[4] Beck CT. A meta-analysis of predictors of postpartum depression. Nurs Res 1996;45(5): 297-303.

[5] Beck CT. The effects of postpartum depression on child development: a metaanalysis. Arch Psychiatr Nurs 1998;12(1):12-20.

[6] Posmontier B, Horowitz JA. Postpartum practices and depression prevalences: technocentric and ethnokinship cultural perspectives. J Transcult Nurs 2004;15(1): 34-43.

[7] Pillsbury BL. "Doing the month": confinement and convalescence of Chinese women after childbirth. Soc Sci Med 1978;12(1B):11-22.

[8] Cheung NF, Mander R, Cheng L, Chen VY, Yang XQ, Qian HP, et al. 'Zuoyuezi' after caesarean in China: an interview survey. Int J Nurs Stud Feb 2006:43(2):193-202.

[9] Heh SS, Coombes L, Bartlett H. The association between depressive symptoms and social support in Taiwanese women during the month. Int J Nurs Stud 2004;41(5): 573-9.

[10] Hung $\mathrm{CH}$, Chung $\mathrm{HH}$. The effects of postpartum stress and social support on postpartum women's health status. J Adv Nurs 2001;36(5):676-84.

[11] Lee DT, Yip AS, Leung TY, Chung TK. Ethnoepidemiology of postnatal depression. Prospective multivariate study of sociocultural risk factors in a Chinese population in Hong Kong. Br J Psychiatry 2004;184:34-40.

[12] Guo SF, Postpartum depression [in Chinese]. Zhonghua Fu Chan Ke Za Zhi $1993 ; 28(9): 532-3,569$.

[13] Xu FS, Liu JX, Zhang SP, Li J, Su Q. Effects of intervening measures on postpartum depression. [in Chinese]. Zhonghua Fu Chan Ke Za Zhi 2003;38(12):724-6.

[14] Zhang R, Chen Q, Li Y. Study for the factors related to postpartum depression [in Chinese]. Zhonghua Fu Chan Ke Za Zhi 1999;34(4):231-3. 
[15] He J, Shangguan X, Wang Z. Clinical study on postpartum depression and its related factors [in Chinese]. Zhonghua Fu Chan Ke Za Zhi 2000;35(11):660-3.

[16] Lee DT, Yip SK, Chiu HF, Leung TY, Chan KP, Chau IO, et al. Detecting postnata depression in Chinese women. Validation of the Chinese version of the Edinburgh Postnatal Depression Scale. Br J Psychiatry 1998;172:433-7.

[17] Xie RH, He G, Liu A, Bradwejn J, Walker M, Wen SW. Fetal gender and postpartum depression in a cohort of Chinese women. Soc Sci Med 2007;65(4):680-4.

[18] The World Bank. China Quick Facts; 2006. Available at: http://web.worldbank.org WBSITE/EXTERNAL/COUNTRIES/EASTASIAPACIFICEXT/CHINAEXTN/0, content MDK:20680895 pagePK:1497618 piPK:217854 theSitePK:318950,00.html Accessed September 30, 2008.

[19] Teng HW, Hsu CS, Shih SM, Lu ML, Pan JJ, Shen WW. Screening postpartum depression with the Taiwanese version of the Edinburgh Postnatal Depression scale. Compr Psychiatry 2005;46(4):261-5.

[20] Pearson V, Phillips MR, He F, Ji H. Attempted suicide among young rural women in the People's Republic of China. Suicide Life Threat Behav 2002;32(4):359-69.
[21] Hoffman S, Hatch MC. Stress, social support and pregnancy outcome: a reassessment based on recent research. Paediatr Perinat Epidemiol 1996;10(4):380-405.

[22] Uddenberg N, Nilsson L. The longitudinal course of para-natal emotional disturbance. Acta Psychiatr Scand 1975;52(3):160-9.

[23] Patel RR, Murphy DJ, Peters TJ. Operative delivery and postnatal depression: a cohort study. BMJ 2005;330(7496):879.

[24] Ware NC, Kleinman A. Culture and somatic experience: The social course of illness in neurasthenia and chronic fatigue syndrome. Psychosom Med 1992·54(5):546-60.

[25] Cheung FM. Conceptualization of psychiatric illness and help-seeking behavior among Chinese. Cult Med Psychiatry 1987;11(1):97-106.

[26] Xu L, Wang Y, Collins CD, Tang S. Urban health insurance reform and coverage in China using data from National Health Services Surveys in 1998 and 2003. BMC Health Serv Res 2007;7:37. 